\title{
The Mechanism of Checkpoint Inhibitors in Gynecologic Cancer
}

\author{
Karoutsos Petros ${ }^{1}$, Karoutsos Dimitrios ${ }^{2}$ and Karoutsou Eftychia ${ }^{3^{*}}$ \\ ${ }^{1}$ Gynecologist, Omilos latrikou Athinon, Athens, Greece \\ ${ }^{2}$ Gynecologist, Department of Obstetrics and Gynecology, General Hospital of Rethymno, Crete, Greece \\ ${ }^{3} \mathrm{MD}$ Endocrinologist
}

*Corresponding author: Karoutsou Eftychia, MD Endocrinologist, Tel: 2130388941; E-mail: eutuxiakaroutsou@yahoo.gr

Received date: December 14, 2016; Accepted date: January 09, 2017; Published date: January 13, 2017

Copyright: (c) 2017 Petros K, et al. This is an open-access article distributed under the terms of the Creative Commons Attribution License, which permits unrestricted use, distribution, and reproduction in any medium, provided the original author and source are credited.

\section{Abstract}

Currently, immunomodulators are used in different cancer entities, including gynecologic malignancies. Toxicity is variable but of narrow extent. Among immunomodulators, checkpoint inhibitors are used to enhance the immune system and significantly improve therapeutic results of advanced disease, mounting tumor progression.

Keywords: Immunomodulators; Immune system; Gynecologic malignancies

\section{Introduction}

The effect of immunotherapy in cancer therapeutics has been identified beforehand via the induction of infection on tumour regression. Coley showed that streptococcus inoculation resulted in the shrinkage of soft-tissue tumors, such as sarcomas. However, results were not verified towards research progress [1].

Paul Ehrlich presented of increased significance that the augmented immune response attacks tumor cells, while tumor cells deceive the immune system promoting tumor growth [2]. For example, interferon$\alpha$ and interleukin-2, have been administrated in advanced melanoma and renal cell carcinoma [3]. Evidence of durable responses in a small proportion of treated patients there exists, suggesting the potential for long-term use with view to survival $[4,5]$; however, these therapeutic modalities appear to be unspecific followed by 'immune related adverse events', making their use of limited scope [6,7].

\section{Immunomodulators - The Example of Ipilimumab}

Ipilimumab has been already used since 2011 for the treatment of melanoma $[8,9]$. It has also been approved for the treatment of nonsmall cell lung carcinoma [10], bladder cancer [11] and metastatic prostate cancer [12].

It has recently been recognized that established tumors evade the immune response by producing inhibitory cytokines, drafting immune cells, and resetting of coinhibitory receptors known as immune checkpoints; immune checkpoint inhibitors, present with completely different mechanism from the conventional anti-neoplastic agents used extensively such as cytotoxic anticancer agents. Therefore, although oncologists expected the therapeutic results to level off, long-term survival or durable response to treatment and toxicity tees towards autoimmune response are currently being of research available.

Immune checkpoints are regulators of the immune system, playing a key role in maintaining self-tolerance, preventing autoimmunity and protecting tissues from immune collateral damage. These immune checkpoints are often 'seized' by tumors to restrain the ability of the immune system to mount an effective anti-tumor response. Blocking immune checkpoints is thus a promising therapeutic approach for 'turning on' the anti-tumor immune response. Many of these pathways are initiated by ligand-receptor interactions on the surface of immune cells, forming targets for monoclonal antibodies. Cytotoxic Tlymphocyte-associated protein-4 (CTLA-4) and programmed cell death protein-1 (PD-1) were the first, and remain the most appropriate immune-checkpoint receptors to be clinically targeted [13]. Although PD-1 and CTLA- 4 belong to the same CD28 family of T-cell receptors, they present very different roles in the down regulation of an inflammatory response. While CTLA- 4 predominantly regulates T cell activation within secondary lymphoid organs, PD-1 predominantly regulates $\mathrm{T}$ cell effector function within peripheral tissues.

CTLA-4 is expressed on the surface of naive effector T cells and regulatory $\mathrm{T}$ cells (Tregs) [14]. When naive $\mathrm{T}$ cells are stimulated, CTLA-4 is upregulated and competes with CD28 for B7 and, finally, determines the suppression of $\mathrm{T}$ cell activity [15]. It was found that the antitumor effect of CTLA-4 blockade might be obtained also by depletion of Treg [16], as revealed in a model of mouse melanoma.

On the contrary, PD-1 is expressed on for a long term periodstimulated T cells, as well as Tregs, activated B cells, and NK cells [17]. Differently from CTLA-4, which regulates T-lymphocytes at the level of initial activation, PD-1 is a key immune-checkpoint receptor expressed by activated $\mathrm{T}$ cells, and it mediates immunosuppression. PD-1 functions primarily in peripheral tissues, where $\mathrm{T}$ cells may encounter the immunosuppressive PD-1 ligands PD-L1 and PD-L2, which are expressed by tumor cells, stromal cells, or both. Inhibition of the interaction between PD-1 and PD-L1 can enhance antitumor activity [18].

PD-1 deficient mice present with enhanced immunity with immune disorders, such as autoimmune cardiomyopathy and lupus erythematosus [19]; both CTLA-4 and PD-1 function as negative regulators which attenuate normal T-cell activation to prevent pathologic over-activation (Figure 1). 


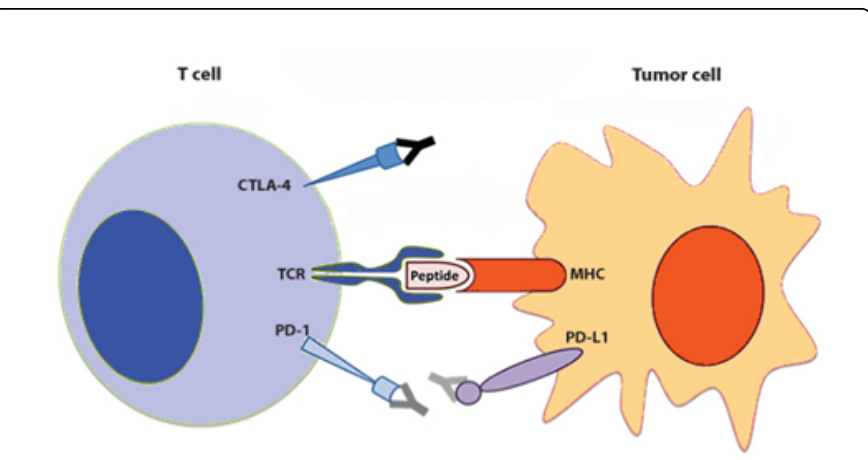

Figure 1: Schematic representation of check point inhibition.

Unfortunately, not all patients respond to these therapies, and evaluation of biomarkers associated with clinical outcomes is further needed [20]. Actually, the immune response plays an important role in the progression of gynecologic malignancies, and preliminary results exhibit the presence of activity of immune checkpoint inhibitors in ovarian, endometrial, and cervical cancer. Now that progression-free survival has come to be an increasingly important trial endpoint, the criteria that define progression deserve critical evaluation to determine whether alternate definitions of progression might facilitate tumor monitoring [21].

\section{Gynecologic Cancer}

Cancer of the ovary is the most common of this group, with an incidence rate of 20 per 100000 women; it also has the poorest prognosis. One third of women survive for five years, compared with over two thirds of those diagnosed with endometrial or cervical cancer. With an ageing population, the proportion of women over the age of 65 with cancer is expected to rise substantially over the next decade [22]. Ovarian and endometrial cancer is more common in older women, whereas the incidence of cervical cancer varies little with age among women over 30 .

\section{Ovarian cancer}

On administering IL-2 intraperitoneally in patients with persistent or recurrent epithelial ovarian cancer, an overall response rate of $25.7 \%$ was shown, though the regimen was associated with significant toxicity. The use of IL-12-expressing plasmids-has been explored. In a recent study, 22 patients with recurrent epithelial ovarian cancer who received intraperitoneal EGEN-001, an IL-12 plasmid formulated with lipopolymer, demonstrated a $35 \%$ stable disease rate [23].

Hodi et al. firstly showed [24,25] antitumor effects in patients with stage IV ovarian cancer by using checkpoint inhibitors. Initially, they reported that a single infusion of ipilimumab $(3 \mathrm{mg} / \mathrm{kg})$ in two-stage IV ovarian cancer patients previously vaccinated with granulocytemacrophage colony-stimulating factor modified irradiated autologous tumor cells (GVAX), was well tolerated, and expedited a decrease or stabilization of CA-125 levels of several months' duration.

The combination of CTLA-4 and PD-1 blockade has been associated with synergistic activity in animal models. A recent phase III study evaluating combined CTLA-4 and PD-1 blockade (with ipilimumab and nivolumab, respectively) in patients with melanoma demonstrated enhanced response rate and progression-free survival compared with either agent alone, leading to recent approval of the combination for the treatment of melanoma [26], although the regimen resulted in high rates of grade 3 toxicity.

Regarding the ovarian cancer presenting with strong immunogenicity, immune checkpoint inhibitors are being evaluated in the parameter of overcoming the 'turn off' signals delivered by the tumor. The latter one having had already been adopted a jester role against the immune system [27]. Several antagonists of PD-1 and PDL1 have been tested in ovarian cancer. These drugs are generally well tolerated, with some immune-related adverse events [28].

Tremelimumab is a fully human monoclonal IgG2 antibody against CTLA-4. In a Phase III trial in patients with advanced melanoma, tremelimumab did not show a statistically significant increase in survival rate over standard chemotherapy [29]. Currently, there is an ongoing Phase I study to evaluate the combination of tremelimumab and a PD-1 inhibitor for patients with ovarian or cervical cancer [30].

A Phase II clinical trial of nivolumab in patients with platinumresistant recurrent ovarian cancer was carried. Unfortunately, there were patients with disease progression taken off this trial [31].

Pembrolizumab is a humanized monoclonal antibody against PD-1 with similar anti-tumor properties as nivolumab for several solid tumors, including melanoma and non-small cell lung cancer [32,33]. Results recently became available from a multi-cohort Phase Ib clinical trial of pembrolizumab for PD-L1 solid tumors including 26 patients with recurrent ovarian cancers [34]. Patch et al. recently showed that germline BRCA1 mutation-associated ovarian cancer has a higher mutational load and quantity of neoantigens compared with BRCA wild-type ovarian cancer [35]. Patients with these types of ovarian cancer are appropriate for therapy with PD-1/PD-L1 inhibition.

Adoptive cell therapies depend upon the infusion of large numbers of autologous tumor-reactive $\mathrm{T}$ cells that have been isolated from tumors and expanded in vitro. Early studies reported success for this approach in epithelial ovarian cancer [36], although these studies were given a bias to by selection of patients from whom a sufficient quantity of tumor-infiltrating lymphocytes could be isolated.

\section{Cervical Cancer}

Live vector-based vaccines are particularly effective, as they deliver antigen efficiently and can replicate within the cell. Live vector-based vaccines can be subdivided into those that use bacterial vectors and those that use viral vectors. The bacterial vector for HPV-associated cancers that is most advanced in clinical development is Listeria monocytogenes.

A prospective phase II study using Listeria vector with and without cisplatin chemotherapy was conducted in patients with recurrent cervical cancer previously treated with chemotherapy, radiotherapy, or both. The results, presented at the American Society of Clinical Oncology 2014 Annual Meeting, showed a 12-month survival rate of $36 \%$; an 18 -month survival rate of $28 \%$; and an $11 \%$ response rate, which was independent of the use of cisplatin [37]. Antibody modulation of T-cell coinhibitory (e.g., CTLA-4) or costimulatory (e.g., 4-1BB) receptors promotes clinical responses to a variety of cancers. Therapeutic cancer vaccination, in contrast, has produced limited clinical benefit and no curative therapies. Monadic potential of $4-1 \mathrm{BB}$ agonist antibody to promote durable regression of HPV+ tumors when combined with an E6/E7 peptide vaccine [38]. 
The therapeutic effects of HPV vaccines may be further augmented by combination with blocking the factors that inhibit $\mathrm{T}$ cell activation, such as CTLA-4 and PD-1, prolonging antitumoral $\mathrm{T}$ cell responses [39]. The combination of HPV therapeutic vaccines with agents that influence the tumor microenvironment may also potentially be used to generate enhanced therapeutic effects against HPV-associated malignancies. It is now clear that several factors present in the tumor microenvironment may potentially inhibit immunotherapy.

The available evidence display that both active and adoptive immunotherapeutical strategies are quite effective against small tumor burdens, but are usually insufficient to eradicate the disease in patients with advanced stages of different kinds of cancer, despite strong induction of tumor-specific immune responses. Although chemotherapy and immunotherapy have not shown to be curative as single modalities, cumulative evidence suggests that combination of these treatments holds potential for improved clinical outcomes in advanced stages of cancer. Therefore, the combination of chemotherapy and immunotherapy is no longer considered incompatible, because of the emerging insight that certain chemotherapy-based cancer treatments may activate the immune system against the tumor through several molecular and cellular mechanisms [40].

A phase I trial of ipilimumab is currently undergoing in patients with locally advanced cervical cancer. Also, a phase II trial is currently testing the efficacy of nivolumab in treating patients with persistent, recurrent, or metastatic cervical cancer (NCT02257528).

As already mentioned, other cells and molecules in the tumor microenvironment [41] other than check point inhibitors that have immune inhibiting functions include regulatory $\mathrm{T}$ cell, MDSC, indoleamine 2, 3-dioxygenase, arginase, nitric oxide synthase, IL-4induced gene 1, IL-10, and TGF- $\beta$. The ability to overcome this immune suppression may further enhance the potency of immunotherapy against HPV-associated cancers.

\section{Endometrial cancer}

Vanderstraeten et al. described PD-L1 expression levels of 67-100\% in primary, recurrent and metastatic endometrial cancer specimens [42]. At the 2015 annual meeting of the Society of Gynecologic Oncology, Herzog et al. reported PD-1 expression levels of $75 \%$, and PD-L1 expression levels ranging from 25-47\%, once again surpassing all examined cervical and ovarian cancer specimens [43]. Given the above, the research of immune checkpoint inhibitors in patients with metastatic and recurrent endometrial cancer may represent a promising alternative to conventional cytotoxic agents.

The best argument for the use of checkpoint inhibitors in select endometrial cancer cases was recently put forth by a phase 2 trial of pembrolizumab, a humanized monoclonal antibody to the PD-1 receptor [44]. Recent knowledge about the microenvironment in endometrial cancer and what has been learned from preliminary immunotherapy trials that enrolled endometrial cancer patients, applauses further attempts at immunomodulation in the treatment of aggressive forms of this disease [45].

Howitt et al. specifically examined the hypothesis that microsatellite unstable endometrial cancers would exhibit more tumor specific neoantigens, resulting in increased tumor infiltrating lymphocytes and a compensatory up-regulation of immune checkpoints [46,47]. Microsatellite unstable tumors exhibited higher numbers of tumor infiltrating lymphocytes. Furthermore, PD-1 was overexpressed in tumor infiltrating lymphocytes, and peri-tumoral lymphocytes of microsatellite unstable tumors.

\section{Endocrine disorders with checkpoint inhibitor immunotherapy for gynecologic cancer}

Endocrinopathies is the aftereffect of immune infiltration into either the thyroid or pituitary glands, resulting in thyroiditis or hypophysitis, which might occur as hypothyroidism or hypopituitarism, including adrenal insufficiency and hypogonadism. Patients who present with new episodes of severe headache need to be urgently assessed with MRI of the pituitary gland. The diagnosis of hypophysitis is put forward by the illustrated enlargement of the pituitary gland and stalk on the MRI. Thyroid function should be assessed before each dose of ipilimumab, with additional endocrine assessments of the anterior lobe of the pituitary as clinically indicated,as well as of the posterior one [47].

Clinically significant endocrinopathy typically is thought to occur in less than 10 percent of patients treated with CTLA-4 blockade [48]. On reviewing the data of immune checkpoint-induced hypophysitis for CTLA-4 drugs, the incidence of hypophysitis for ipilimumab was 0$17 \%,(48,49)$ while that for tremelimumab (phase 1 and randomized clinical trial data) was $0.4-5 \%[49,50]$. The incidence was relatively less for other immune checkpoint inhibitors such as nivolumab [51].

Overall, the endocrine effects of these drugs include hypophysitis and thyroid dysfunction, with rare reports of adrenal hypofunction. Primary thyroid dysfunction occurs in up to $15 \%$ of patients, with adrenalitis reported in approximately only $1 \%$. The mean onset of endocrine side effects is 9 weeks after initiation (range 5-36 weeks) (49). Hypothyroidism was reported in approximately $2 \%$ of patients treated with ipilimumab and up to $8.3 \%$ of patients with treated with PD-1 inhibitors; the time to onset ranged from 0.7 weeks to 19 months in PD-1 inhibitor trials [52,53].

\section{Thyroid Disorders}

Destructive thyroiditis or Graves disease are the main causes of thyroid hypo- or hyper- function respectively [54,55]. According to Common Terminology Criteria for Adverse Events (CTCAE) the toxicities of hypothyroidism and hyperthyroidism encountered ranges from grade 1 to grade 2 toxicities [56]. Up till now, clinical data for both CTLA- 4 and PD-1 blockade suggest that the incidence of primary hyperthyroidism is being lower than primary hypothyroidism.

Hyperthyroidism and hypothyroidism have also been described in patients treated with pembrolizumab and nivolumab [57]. The most frequently observed immune related adverse events related to thyroid function are hypothyroidism, thyroiditis, hyperthyroidism, which usually range from asymptomatic to moderate symptoms presentation that is CTCAE grade 1 or 2 toxicity, respectively [32].

Management of primary hypothyroidism, regarding grade 2 toxicity for CTCAE, involves replacement with thyroid hormone, levothyroxine, and endocrinologic consultation. Hyperthyroidism may represent acute thyroiditis secondary to immune activation for which a short period of high-dose steroids ( $1 \mathrm{mg} / \mathrm{kg}$ prednisone or equivalent) may need to be considered for symptomatic patients. Most patients subsequently become hypothyroid and need long-term hormone replacement [58]. Hypothyroidism is diagnosed if TSH level is increased with a low free T4 level, whereas hypophysitis with central 
hypothyroidism, presents with a low TSH and low free T4. Immune checkpoint inhibitor therapy may be continued without interruption with appropriate levothyroxine replacement $[59,60]$.

\section{Pituitary Disorders}

Pituitary enlargement indicates precisely hypophysitis in patients being receiving ipilimumab. In fact, relative radiographic enlargement of the pituitary preceded the diagnosis of hypophysitis. The next step is overall evaluation that much of the anterior pituitary lobe as of the posterior, in any patient being administered ipilimumab who exhibits gross pituitary enlargement or even a relative one in imaging studies that is MRI.

Although pituitary enlargement seems to be a sensitive indicator for hypophysitis, is not recommended that clinicians obtain routine MRIs in asymptomatic patients treated with ipilimumab because most patients do not develop hypophysitis. On the other hand [59], thyroid function tests are routinely measured before each ipilimumab treatment cycle. Central hypothyroidism in all patients with hypophysitis in recent observations denotes that CTLA-4 is expressed preferentially by thyrotropin-secreting cells in the anterior pituitary [61].

Actually, it can present insidiously and have life-threatening complications related to hypocortisolism that is grade 4 toxicity as to CTCAE, hence it should be clinically suspected. Improvement of symptoms and resolution of radiological findings are possible with early treatment with steroids and hormone replacement therapy of deficiency seperately identified [62].

"An immediate implication for patients is that they can now be tested for the presence of pituitary antibodies to diagnose the presence or predict the development of hypophysitis," explained author Patrizio Caturegli, MD, from Johns Hopkins University in Baltimore.

"A down-the-line application is for pharmaceutical companies to make an antibody that does not bind complement - for example, changing the isotype from IgG1 (like the isotype of ipilimumab) to IgG4 (an isotype that does not have complement binding capacity), or engineering the antibody molecule to remove the complement binding motif," Dr. Caturegli described. However, predictive factors for onset of autoimmune hypophysitis remain unclear and it is imperative that this entity is differentially diagnosed from pituitary metastases.

Furthermore, most male patients had hypogonadotropic hypogonadism that is only grade 1 toxicity for CTCAE. In a case series study [63], 3 out of 5 patients had low serum IGF-I. Prolactin levels may be elevated or low in approximately $25 \%$ of patients $[64,65]$. Only one patient had been diagnosed with diabetes insipidus [66]. Treatment recommendation for hypophysitis is methylprednisolone $125 \mathrm{mg}$ intravenously daily or dexamethasone $6 \mathrm{mg}$ every 6 hours intravenously for 3 days with a switch to oral prednisone $1-2 \mathrm{mg} / \mathrm{kg}$ daily after improvement of symptoms. Immune checkpoint inhibitor therapy should be permanently discontinued for severe or lifethreatening grade 3 or 4 toxicity [67].

Severe or life-threatening secondary adrenal insufficiency (grade 4 toxicity for CTCAE) is also included. It is characterized by hypotension, dehydration, hyponatremia, and hyperkalemia imitating septic syndrome. The incidence of severe or life-threatening hypopituitarism is reported in $<2 \%$ of patients being treated with immune checkpoint inhibitors [67]. Adrenal insufficiency requires transfer to the urgency department and management with fluids and corticosteroids intravenously. Corticosteroids should be initiated at 60-80 mg prednisone daily or equivalent and tapered over 1 month. Therefore, endocrine adverse events (e.g., hypothyroidism and adrenal insufficiency) are managed with replacement therapy, and patients reinitiate treatment, for instance with anti-PD-L1 antibody, at the discretion of the treating physician [68] as to grade 2 toxicity for CTCAE.

Rare side-effects included diabetes mellitus, lichen planus, and pancreatic insufficiency due to pancreatitis [69]. Hepatitis, and one case each of sarcoidosis, endophthalmitis and myasthenia gravis have been observed, too [69].

\section{Depression in cancer as a result of immunotherapy}

Depression continues to be underdiagnosed in cancer patients, more pronounced this phenomenon is in the case of immunomodulators prescription.

Treatment-related risk factors is immunotherapy with interferon and IL-2. There is an association between immune activation and early depressive symptoms in cancer patients treated with IL-based therapy [70]. In general, it could be that depression is associated with the decreased cytotoxic T-cell and natural-killer-cell activities that affect processes such as immune surveillance of tumours.

Depression emerges on the background of sickness when the inflammatory response is too intense and long lasting or the resolution process is deficient. The transition from sickness to depression is mediated by activation of the kynurenine metabolic pathway that leads to the formation of neurotoxic metabolites. The neuroimmune processes and molecular factors that have been identified in the studies of inflammation-associated depression introduce potential new targets for the development of advanced therapies for the treatment of major depressive disorders [71]. It is suggested that symptoms of depression would fluctuate over time, depending on the degree of inflammation and kynurenine metabolite levels in the cerebrospinal fluid [72].

As to cancer patients, gene expression studies, in the future, may have the potential to detect changes due to differences in common or rare genomic sequence variation, environmental factors or their interaction. Although cause-effect relationships cannot be determined from these data, the results support the hypothesis that altered immune signaling has a role in the pathogenesis, manifestation, and/or the persistence and progression of major depression. Type I interferon signaling genes in recurrent major depression reveal increased expression detected by whole-blood RNA sequencing [73].

Another mechanism for pro-inflammatory cytokines, particularly for TNF- $\alpha$, is the increase in the activity of an enzyme called indoleamine-2,3-dioxygenase, which degrades tryptophan. Tryptophan is the precursor to 5-hydroxytryptamine; therefore its destruction results in reduced 5-hydroxytryptamine levels [74]. It is well established that tryptophan levels are decreased relative to monoamine levels in the brains of depressed patients [75].

On the other hand, therapeutic agents targeting specific cytokine molecules, such as interleukin-6 or tumor necrosis factor-alpha, are currently being evaluated for their potential to simultaneously treat both depression and cachexia. The available data suggest a dual role for cytokines in the development of cancer-related depression and cachexia [76]. A better understanding of the bidirectional communication between the neuroendocrine and the immune system could contribute to new clinical and treatment strategies with reference 
Page 5 of 6

to patients receiving immune-based therapy for tumors, including those suffering from gynecologic cancer.

\section{Conclusion}

Anti-programmed cell death receptor-1 (PD-1) antibodies represent an effective treatment option for gynecologic cancer as well as for other cancer entities. This review focuses on the mechanism of action of checkpoint inhibitors as to treatment thus much with anti-PD-1 antibodies (nivolumab or pembrolizumab) as with anti-cytotoxic Tlymphocyte antigen-4 inhibition (ipilimumab) in patients with advanced gynecologic malignancies. A better understanding of the management of the adverse events, including endocrine ones, will help ensure the safe and appropriate use of immune-based therapy in gynecologic cancer and other tumor types.

\section{References}

1. Coley WB (1891) Contribution to the knowledge of sarcoma. Ann Surg 14: 199-220.

2. Dunn GP, Bruce AT, Ikeda H, Old LJ, Schreiber RD (2002) Cancer immunoediting: from immunosurveillance to tumor escape. Nat Immunol 3: 991-998.

3. Waldmann TA (2003) Immunotherapy: past, present and future. Nat Med 9: 269-277.

4. Rosenberg SA, Yang JC, White DE, Steinberg SM (1998) Durability of complete responses in patients with metastatic cancer treated with highdose interleukin-2: Identification of the antigens mediating response. Ann Surg 228: 307-319.

5. Rosenberg SA (2012) Raising the bar: the curative potential of human cancer immunotherapy. Sci Transl Med 4: 127ps8.

6. Amin A, White RL Jr (2013) High-dose interleukin-2: is it still indicated for melanoma and RCC in an era of targeted therapies? Oncology (Williston Park) 27: 680-691.

7. Antony GK, Dudek AZ (2010) Interleukin 2 in cancer therapy. Curr Med Chem 17: 3297-3302.

8. Lacroix M (2014) Targeted Therapies in Cancer. Hauppauge, NY: Nova Sciences Publishers.

9. Pollack A (2015) New Class of Drugs Shows More Promise in Treating Cancer. New York Times.

10. ClinicalTrials.gov (2012) Phase II Study for Previously Untreated Subjects With Non Small Cell Lung Cancer (NSCLC) or Small Cell Lung Cancer (SCLC).

11. ClinicalTrials.gov (2012) First-Line Gemcitabine, Cisplatin + Ipilimumab for Metastatic Urothelial Carcinoma.

12. ClinicalTrials.gov (2006) Phase I/II Study of MDX-010 in Patients With Metastatic Hormone-Refractory Prostate Cancer.

13. Pardoll DM (2012) The blockade of immune checkpoints in cancer immunotherapy. Nat Rev Cancer 12: 252-264.

14. Peggs KS, Quezada SA, Chambers CA, Korman AJ, Allison JP (2009) Blockade of CTLA-4 on both effector and regulatory T cell compartments contributes to the antitumor activity of anti-CTLA-4 antibodies. J Exp Med 206: 1717-1725.

15. Linsley PS, Bradshaw J, Greene J, Peach R, Bennett KL, et al. (1996) Intracellular trafficking of CTLA-4 and focal localization towards sites of TCR engagement. Immunity 4: 535-543.

16. Takahashi T, Tagami T, Yamazaki S (2000) Immunologic self-tolerance maintained by CD25+CD4+ regulatory $\mathrm{T}$ cells constitutively expressing cytotoxic T lymphocyte-associated antigen 4. J Exp Med 192: 303-310.

17. Page DB, Postow MA, Callahan MK, Allison JP, Wolchok JD (2014) Immune modulation in cancer with antibodies. Annual Review of Medicine 65: 185-202.
18. Topalian SL, Hodi FS, Brahmer JR, Gettinger SN, Smith DC, et al. (2012) Safety, activity, and immune correlates of anti-PD-1 antibody in cancer. N Engl J Med 366: 2443-2454.

19. Nishimura H, Okazaki T, Tanaka Y, Nakatani K, Hara M, et al. (2001) Autoimmune dilated cardiomyopathy in PD-1 receptor-deficient mice. Science 291: 319-322.

20. Kyi C, Postow MA (2014) Checkpoint blocking antibodies in cancer immunotherapy. FEBS Lett 588: 368-376.

21. Oxnard GR, Morris MJ, Hodi FS, Baker LH, Kris MG, et al. (2012) When progressive disease does not mean treatment failure: reconsidering the criteria for progression. J Natl Cancer Inst 104: 1534-1541.

22. Dumas L, Ring A, Butler J, Kalsi T, Harari D, et al. (2016) Improving outcomes for older women with gynaecological malignancies. Cancer Treat Rev 50: 99-108.

23. Alvarez RD, Sill MW, Davidson SA, Muller CY, Bender DP, et al. (2014) A phase II trial of intraperitoneal EGEN-001, an IL-12 plasmid formulated with PEG-PEI-cholesterol lipopolymer in the treatment of persistent or recurrent epithelial ovarian, fallopian tube or primary peritoneal cancer: a gynecologic oncology group study. Gynecol Oncol 133: 433-438.

24. Hodi FS, Mihm MC, Soiffer RJ (2003) Biologic activity of cytotoxic T lymphocyte-associated antigen 4 antibody blockade in previously vaccinated metastatic melanoma and ovarian carcinoma patients. Proc Natl Acad Sci U S A 100: 4712-4717.

25. Hodi FS, Butler M, Oble DA (2008) Immunologic and clinical effects of antibody blockade of cytotoxic T lymphocyte-associated antigen 4 in previously vaccinated cancer patients. Proc Natl Acad Sci U S A 105: 3005-3010.

26. Larkin J, Hodi FS, Wolchok JD (2015) Combined Nivolumab and Ipilimumab or Monotherapy in Untreated Melanoma. N Engl J Med 373: 1270-1271.

27. Mittica G, Genta S, Aglietta M (2016) Immune Checkpoint Inhibitors: A New Opportunity in the Treatment of Ovarian Cancer? Int J Mol Sci 17.

28. Emens LA, Kok M, Ojalvo LS (2016) Targeting the programmed cell death-1 pathway in breast and ovarian cancer. Curr Opin Obstet Gynecol 28: 142-147.

29. Ribas A, Kefford R, Marshall MA, Punt CJ, Haanen JB, et al. (2013) Phase III randomized clinical trial comparing tremelimumab with standard-ofcare chemotherapy in patients with advanced melanoma. J Clin Oncol 31: 616-622.

30. Callahan MK, Ott PA, Odunsi K (2014) A phase 1 study to evaluate the safety and tolerability of MEDI4736, an anti-PD-L1 antibody, in combination with tremelimumab in patients with advanced solid tumors. J Clin Oncol 32: TPS3120.

31. Hamanishi J, Mandai M, Ikeda T, Minami M, Kawaguchi A, et al. (2015) Safety and Antitumor Activity of Anti-PD-1 Antibody, Nivolumab, in Patients With Platinum-Resistant Ovarian Cancer. J Clin Oncol 33: 4015-4022.

32. Robert C, Schachter J, Long GV, Arance A, Grob JJ, et al. (2015) Pembrolizumab versus Ipilimumab in Advanced Melanoma. N Engl J Med 372: 2521-2532.

33. Massagué J (2008) TGFbeta in Cancer. Cell 134: 215-230.

34. Varga A, Piha-Paul SA, Ott PA (2015) Antitumor activity and safety of pembrolizumab in patients (pts) with PD-L1 positive advanced ovarian cancer: interim results from a phase Ib study. J Clin Oncol 33: 5510.

35. Patch AM, Christie EL, Etemadmoghadam D, Garsed DW, George J, et al. (2015) Whole-genome characterization of chemoresistant ovarian cancer. Nature 521: 489-494.

36. Fujita K, Ikarashi H, Takakuwa K (1995) Prolonged disease-free period in patients with advanced epithelial ovarian cancer after adoptive transfer of tumor-infiltrating lymphocytes. Clin Cancer Res 1: 501-507.

37. Basu P, Mehta AO, Jain MM (2014) ADXS11-001 immunotherapy targeting HPV-E7: final results from a phase 2 study in Indian women with recurrent cervical cancer. J Clin Oncol 32: 5610.

38. Bartkowiak T, Singh S, Yang G, Galvan G, Haria D, et al. (2015) Unique potential of $4-1 \mathrm{BB}$ agonist antibody to promote durable regression of 
HPV+ tumors when combined with an E6/E7 peptide vaccine. Proc Natl Acad Sci U S A 112: E5290-E5299.

39. Peggs KS, Quezada SA, Korman AJ, Allison JP (2006) Principles and use of anti-CTLA4 antibody in human cancer immunotherapy. Curr Opin Immunol 18: 206-213.

40. Van Meir H, Kenter GG, Burggraaf J, Kroep JR, Welters MJ, et al. (2014) The need for improvement of the treatment of advanced and metastatic cervical cancer, the rationale for combined chemo-immunotherapy. Anticancer Agents Med Chem 14: 190-203.

41. Lee SJ, Yang A, Wu TC, Hung CF (2016) Immunotherapy for human papillomavirus-associated disease and cervical cancer: review of clinical and translational research. J Gynecol Oncol 27: e51.

42. Vanderstraeten A, Luyten C, Verbist G, Tuyaerts S, Amant F (2014) Mapping the immunosuppressive environment in uterine tumors: implications for immunotherapy. Cancer Immunol Immunother 63: 545557.

43. Herzog T, Arguello D, Reddy S, Gatalica Z (2015) PD-1 and PD-L1 expression in 1599 gynecological malignancies - implications for immunotherapy. Gynecol Oncol 137: 1.

44. Le DT, Uram JN, Wang H, Bartlett BR, Kemberling H, et al. (2015) PD-1 Blockade in Tumors with Mismatch-Repair Deficiency. N Engl J Med 372: 2509-2520.

45. Longoria TC, Eskander RN (2015) Immunotherapy in endometrial cancer-an evolving therapeutic paradigm. Gynecol Oncol Res Pract 2: 11.

46. Bender D, Sill MW, Lankes HA, Darus CJ, Delmore JE, Rotmensch J, et al. (2015) A phase II evaluation of cediranib in the treatment of recurrent or persistent endometrial cancer: An NRG Oncology/Gynecologic Oncology Group (GOG) study. Gynecol Oncol 138: 507-512.

47. Gangadhar TC, Vonderheide RH (2014) Mitigating the toxic effects of anticancer immunotherapy. Nat Rev Clin Oncol 11: 91-99.

48. Corsello SM, Barnabei A, Marchetti P, De Vecchis L, Salvatori R, et al. (2013) Endocrine side effects induced by immune checkpoint inhibitors. J Clin Endocrinol Metab 98: 1361-1375.

49. Torino F, Barnabei A, Paragliola RM, Marchetti P, Salvatori R, et al. (2013) Endocrine side-effects of anti-cancer drugs: mAbs and pituitary dysfunction: clinical evidence and pathogenic hypotheses. Eur J Endocrinol 169: 153-164.

50. Ribas A, Camacho LH, Lopez-Berestein G (2005) Antitumor activity in melanoma and anti-self responses in a phase I trial with the anticytotoxic $\mathrm{T}$ lymphocyte-associated antigen 4 monoclonal antibody CP-675, 206. J Clin Oncol 23: 8968-8977.

51. Okano Y, Satoh T, Horiguchi K, Toyoda M, Osaki A, et al. (2016) Nivolumab-induced hypophysitis in a patient with advanced malignant melanoma. Endocr J 63: 905-912.

52. Robert C, Ribas A, Wolchok JD (2014) Anti-programmed-deathreceptor-1 treatment with pembrolizumab in ipilimumab-refractory advanced melanoma: a randomised dose-comparison cohort of a phase 1 trial. Lancet 384: 1109-1117.

53. Rizvi NA, Mazières J, Planchard D (2015) Activity and safety of nivolumab, an anti-PD-1 immune checkpoint inhibitor, for patients with advanced, refractory squamous non-small-cell lung cancer (CheckMate 063): a phase 2, single-arm trial. Lancet Oncol 16: 257-265.

54. https://www.hcp.yervoy.com/pdf/rems-management-guide.pdf.

55. http://www.merck.com/product/usa/pi_circulars/k/keytruda/ keytruda_pi.pdf

56. Common Terminology Criteria for Adverse Events (CTCAE) (2010) Version 4.0, U.S. Department of Health and Human Services, National Institutes of Health, National Cancer Institute.

57. http://packageinserts.bms.com/pi/pi_opdivo.pdf.

58. Weber JS, Kähler KC, Hauschild A (2012) Management of immunerelated adverse events and kinetics of response with ipilimumab. J Clin Oncol 30: 2691-2697.
59. Yervoy (2013) Bristol-Myers Squibb, package insert. Princeton, NJ: Bristol-Myers Squibb.

60. Fecher LA, Agarwala SS, Hodi FS, Weber JS (2013) Ipilimumab and its toxicities: a multidisciplinary approach. Oncologist 18: 733-743.

61. Iwama S, De Remigis A, Callahan MK, Slovin SF, Wolchok JD, et al. (2014) Pituitary expression of CTLA-4 mediates hypophysitis secondary to administration of CTLA-4 blocking antibody. Sci Transl Med 6: $230 \mathrm{ra} 45$.

62. Lam T, Chan MM, Sweeting AN (2015) Ipilimumab-induced hypophysitis in melanoma patients: an Australian case series. Intern Med J 45: 1066-1073.

63. Min L, Vaidya A, Becker C (2012) Association of ipilimumab therapy for advanced melanoma with secondary adrenal insufficiency: a case series. Endocr Pract 18: 351-355.

64. Blansfield JA, Beck KE, Tran K (2005) Cytotoxic T-lymphocyte-associated antigen- 4 blockage can induce autoimmune hypophysitis in patients with metastatic melanoma and renal cancer. J Immunother 28: 593-598.

65. Maker AV, Yang JC, Sherry RM, Topalian SL, Kammula US, et al. (2006) Intrapatient dose escalation of anti-CTLA- 4 antibody in patients with metastatic melanoma. J Immunother 29: 455-463.

66. Dillard T, Yedinak CG, Alumkal J, Fleseriu M (2010) Anti-CTLA-4 antibody therapy associated autoimmune hypophysitis: serious immune related adverse events across a spectrum of cancer subtypes. Pituitary 13: 29-38.

67. Villadolid J, Amin A (2015) Immune checkpoint inhibitors in clinical practice: update on management of immune-related toxicities. Transl Lung Cancer Res 4: 560-575.

68. Brahmer JR, Tykodi SS, Chow LQ, Hwu WJ, Topalian SL, et al. (2012) Safety and activity of anti-PD-L1 antibody in patients with advanced cancer. N Engl J Med 366: 2455-2465.

69. Hofmann L, Forschner A, Loquai C, Goldinger SM, Zimmer L, et al. (2016) Cutaneous, gastrointestinal, hepatic, endocrine, and renal sideeffects of anti-PD-1 therapy. Eur J Cancer 60: 190-209.

70. Capuron L, Ravaud A, Gualde N, Bosmans E, Dantzer R, et al. (2001) Association between immune activation and early depressive symptoms in cancer patients treated with interleukin-2-based therapy. Psychoneuroendocrinology 26: 797-808.

71. Dantzer R (2017) Role of the Kynurenine Metabolism Pathway in Inflammation-Induced Depression: Preclinical Approaches.Curr Top Behav Neurosci 31: 117-138.

72. Bay-Richter C, Linderholm KR, Lim CK, Samuelsson M, TräskmanBendz L, et al. A role for inflammatory metabolites as modulators of the glutamate $\mathrm{N}$-methyl-D-aspartate receptor in depression and suicidality. Brain Behav Immun 43: 110-117.

73. Mostafavi S, Battle A, Zhu X, Potash JB, Weissman MM, et al. (2014) Type I interferon signaling genes in recurrent major depression: increased expression detected by whole-blood RNA sequencing. Mol Psychiatry 19: 1267-1274.

74. Dantzer R, O'Connor JC, Freund GG, Johnson RW, Kelley KW (2008) From inflammation to sickness and depression: when the immune system subjugates the brain. Nat Rev Neurosci 9: 46-56.

75. DeMyer MK, Shea PA, Hendrie HC, Yoshimura NN (1981) Plasma tryptophan and five other amino acids in depressed and normal subjects. Arch Gen Psychiatry 38: 642-646.

76. Illman J, Corringham R, Robinson D Jr, Davis HM, Rossi JF, et al. (2005) Are inflammatory cytokines the common link between cancer-associated cachexia and depression? J Support Oncol 3: 37-50. 